Article

\title{
Aerogels from Chitosan Solutions in Ionic Liquids
}

\author{
Gonzalo Santos-López ${ }^{1}$, Waldo Argüelles-Monal ${ }^{2}$ [D, Elizabeth Carvajal-Millan ${ }^{1}$, \\ Yolanda L. López-Franco ${ }^{1}$ (D), Maricarmen T. Recillas-Mota ${ }^{2}$ and Jaime Lizardi-Mendoza ${ }^{1, *}$ \\ 1 Grupo de Investigación en Biopolímeros-CTAOA. Centro de Investigación en Alimentación y Desarrollo, \\ A.C., Hermosillo, Sonora 83304, Mexico; gonzalosantos@estudiantes.ciad.mx (G.S.-L.); \\ ecarvajal@ciad.mx (E.C.-M.); lopezf@ciad.mx (Y.L.L.-F.) \\ 2 Polímeros Naturales. Centro de Investigación en Alimentación y Desarrollo, A.C., Unidad Guaymas, \\ Guaymas, Sonora 85480, Mexico; waldo@ciad.mx (W.A.-M.); mrecillas@ciad.mx (M.T.R.-M.) \\ * Correspondence: jalim@ciad.mx; Tel.: +52-662-289-2400
}

Received: 16 November 2017; Accepted: 14 December 2017; Published: 16 December 2017

\begin{abstract}
Chitosan aerogels conjugates the characteristics of nanostructured porous materials, i.e., extended specific surface area and nano scale porosity, with the remarkable functional properties of chitosan. Aerogels were obtained from solutions of chitosan in ionic liquids (ILs), 1-butyl-3-methylimidazolium acetate (BMIMAc), and 1-ethyl-3-methyl-imidazolium acetate (EMIMAc), in order to observe the effect of the solvent in the structural characteristics of this type of materials. The process of elaboration of aerogels comprised the formation of physical gels through anti-solvent vapor diffusion, liquid phase exchange, and supercritical $\mathrm{CO}_{2}$ drying. The aerogels maintained the chemical identity of chitosan according to Fourier transform infrared spectrophotometer (FT-IR) spectroscopy, indicating the presence of their characteristic functional groups. The internal structure of the obtained aerogels appears as porous aggregated networks in microscopy images. The obtained materials have specific surface areas over $350 \mathrm{~m}^{2} / \mathrm{g}$ and can be considered mesoporous. According to swelling experiments, the chitosan aerogels could absorb between three and six times their weight of water. However, the swelling and diffusion coefficient decreased at higher temperatures. The structural characteristics of chitosan aerogels that are obtained from ionic liquids are distinctive and could be related to solvation dynamic at the initial state.
\end{abstract}

Keywords: aerogels; chitosan; ionic liquids; ionogels

\section{Introduction}

Chitosan (Cs) is a natural linear polysaccharide generated from the deacetylation of chitin and is composed of $\beta$-(1-4)-D-glucosamine units and $\beta$-(1-4)-N-acetyl-glucosamine distributed along the polymeric chain. The physicochemical characteristics and functional properties of Cs, such as its polycationic character, biocompatibility, low toxicity, and structural capacity, make it a polysaccharide of interest in different fields. The mechanical and structural properties of chitosan allow for different types of materials to be obtained from chitosan solutions, e.g., nanostructured porous materials. Aerogels are a specific type of nanostructured porous material that are characterized by mesopores (pore diameter between 2 and $50 \mathrm{~nm}$ ) and large surface areas. Obtaining aerogels of chitosan could improve the availability of the functional groups of chitosan [1]. Together, the aerogel characteristics with the functional properties of chitosan, potentially provides materials with multiple applications, e.g., adsorption, transport, and controlled release of bioactive molecules, toxics, and pollutants removers, among others.

Ionic liquids (IL) are an alternative medium for chitosan dissolution. When compared to the traditional aqueous acid solvents used to dissolve Cs, IL have different physicochemical characteristics because they consist of only ions and water is not needed to dissolve chitosan. Most of the 
studies of chitosan with IL have been focused on the formation of solutions. Imidazolium based $\mathrm{IL}$, as 1-butyl-3-methylimidazolium acetate (BMIMAc), and 1-ethyl-3-methyl-imidazolium acetate (EMIMAc), have been highlighted because they are able to dissolve chitosan at high concentrations (up to $10 \% w / w$ ). Other reports have focused on forming materials from chitosan solutions in IL e.g., fibers, blends, films, membranes, hydrogels, and ionogels [2-12]. Aerogels from chitin with BMIMAc solutions has been reported [13], but the information about chitosan ionogels and aerogels from IL solutions is limited.

Supercritical $\mathrm{CO}_{2}$ drying, unlike other types of drying, keeps most of the internal structure formed at gelation because the effects of surface tension on the three-dimensional macromolecular network are minimized. Therefore, chitosan aerogels that are generated from solutions of Cs in IL (BMIMAc and EMIMAc) were produced in order to study the physicochemical characteristics of these novel materials. Concurrently, observations on the structural features of the aerogels could be related to the network formation and molecular conformation of chitosan in ionic liquids at the physical gelling process.

\section{Materials and Methods}

\subsection{Materials}

Shrimp shells (Pandalus borealis) Cs was acquired from Primex (batch No. TM 1961, Siglufjörður, Iceland). The degree of acetylation (DA) of Cs was $16.3 \%$, as determined by solid-state cross-polarization magic angle spinning ${ }^{13} \mathrm{C}$ nuclear magnetic resonance spectroscopy (CP/MAS ${ }^{13} \mathrm{C}$-NMR) performed in a Bruker Avance TM 400WB (Bruker Biospin, Wissembourg, France, 9.4 Tesla, $1 \mathrm{~ms}$ contact time, 2000 scans) [14,15]. The weight average molecular weight $\left(M_{\mathrm{W}}\right)$ of $C$ s was $2.01 \times 10^{5} \mathrm{~g} / \mathrm{mol}$, determined by static light scattering, as described previously [16]. Chitosan was purified, as described by Rinaudo et al. (1999), before use it [17]. Commercial grade IL, BMIMAc and EMIMAc (BASF, Steinheim, Germany), were used as received just prior each use they were stored in desiccator and kept at $105^{\circ} \mathrm{C}$ for $24 \mathrm{~h}$ in vacuum to minimize moisture. High grade supercritical drying liquid $\mathrm{CO}_{2}(99.99 \%$ purity) was supplied by Infra (Hermosillo, México). All of the compounds and solvents that were used were reagent grade acquired from recognized commercial chemical distributors. The water used for the experiments was deionized type I (resistivity of $18.2 \mathrm{M} \Omega \cdot \mathrm{cm}$ at $25^{\circ} \mathrm{C}$ ) unless stated otherwise.

\subsection{Solutions of Chitosan in Ionic Liquids}

The moisture-free IL was added into a round-bottom flask with a sufficient amount of chitosan to obtain a $2 \%(w / w)$ concentration. This solution was heated in an oil bath at $105{ }^{\circ} \mathrm{C}$ under magnetic stirring at least 6 hours under a nitrogen atmosphere. Upon complete Cs dissolution it was stored in a desiccator at room temperature until use. Two types of solutions were obtained: Cs in EMIMAc (CsEMIM) and Cs in BMIMAc (CsBMIM).

\subsection{Formation of Ionogels}

The physical gels were formed using approximately $0.1 \mathrm{~g}$ of the Cs-IL solution that was casted in cylindrical containers $(0.4 \mathrm{~cm}$ diameter $)$, with the bottom being covered with plastic paraffin film. Gelation was induced by vapor diffusion of an antisolvent, such as ethanol or water, in a closed chamber [18]. The gels were recovered after $48 \mathrm{~h}$ and were transferred to a $70 \%$ ethanol aqueous mixture. These gels that contain IL as liquid phase confined within a polymer matrix are defined as ionogels [19].

\subsection{Formation of Aerogels}

The ionogels were thoroughly rinsed with aqueous ethanol (70\%) until the IL was completely removed. The presence of IL in the rinsing media was monitored with UV/VIS spectroscopy, scanning between 190 to $300 \mathrm{~nm}$ using the ethanol-water mixture as reference. Afterwards, the liquid phase 
was replaced with acetone, which has higher miscibility with supercritical $\mathrm{CO}_{2}$. For this, the gels were repeatedly rinsed with a gradient of acetone-water mixtures that ended with two rinses with pure acetone. The acetogels (gels containing acetone as fluid phase) were dried with supercritical $\mathrm{CO}_{2}$ $\left(>32{ }^{\circ} \mathrm{C}\right.$ and $\left.73 \mathrm{~atm}\right)$ in a pressurized reactor. At the end of the process, the dried aerogels were stored in a desiccator at room temperature.

\subsection{Characterization of Aerogels}

\subsubsection{Chemical Identity}

The chemical composition of the samples was determined analyzing the characteristic bands in the infrared spectrum obtained by a Fourier transform infrared spectrophotometer (FT-IR, Thermo Scientific, Nicolet iS-50, Madison, WI, USA), using the attenuated total reflection (ATR) mode. All of the measurements were performed at room temperature collecting 32 scans at $4 \mathrm{~cm}^{-1}$ resolution.

\subsubsection{Structural Analysis}

The surface area, pore volume, and nitrogen adsorption and desorption isotherms of the aerogels were determined with the surface area analyzer Nova 2200e (Quantachrome Instruments, Boynton Beach, FL, USA), and analysis of the data with the software NovaWin version 11.02 (Quantachrome Instruments, Boynton Beach, FL, USA). The morphological characteristics of the aerogels were thoroughly observed by field emission scanning electron microscopy (Hitachi SU8000, Tokyo, Japan). The images were obtained using an accelerating voltage of $1.0 \mathrm{KeV}$.

\subsubsection{Degree of Swelling}

The swelling capacity of the aerogels at equilibrium was determined from their immersion in water at different temperatures $\left(20,25,30\right.$, and $\left.40^{\circ} \mathrm{C}\right)$. The weight gain of the samples was periodically monitored by removing the excess of water with filter paper. Equation (1) was used to estimate the degree of swelling $(W)$, as follows:

$$
W=\frac{P-P_{0}}{P_{0}}=\frac{P}{P_{0}}-1
$$

where $P_{0}$ is the weight of the dry aerogel and $P$ is the weight of the aerogel in the wet state [20].

\section{Results and Discussion}

The vapor diffusion of a non-solvent agent was useful to produce ionogels from Cs solutions in IL. By this way, it was possible to obtain three different types of physical chitosan ionogels (Table 1). Using ethanol as non-solvent agent gels were obtained from both Cs-IL solutions. These gels were clear, rigid, and brittle; the only noticeable difference among them was the color tone, darker yellow for the gels from CsBMIM. It has been indicated that the main effect of IL on polysaccharides is to disrupt the hydrogen bonds and promoting their dissolution. Low molecular weight alcohols are miscible with imidazolium based ionic liquids [21], but chitosan does not dissolve in alcohols. Therefore, when ethanol diffuses into a Cs-IL solution, the solvation effect of the ionic liquid over chitosan decreases, favoring the interactions between chitosan chains and subsequently leading to the generation of a gel. Conversely, water vapor was only useful to produce gels from the CsEMIM solution. It has been noticed that BMIMAc has a lower affinity for water than EMIMAc [22], this could be related to lower diffusion rates that do not decrease its chitosan solvation capacity in preventing the gel formation. It should be taken in account that chitosan do not dissolve in water, but it is hygroscopic. As result, the obtained gels from water diffusion were weak and difficult to manipulate without compromising their integrity. Hence, subsequent procedures and analysis were performed using only ionogels that are produced by ethanol treatment. 
Table 1. Outcome of the treatment of Cs-IL solutions with non-solvent agents vapor diffusion.

\begin{tabular}{ccc}
\hline \multirow{2}{*}{ Cs-IL Solution } & \multicolumn{2}{c}{ Non-Solvent Agent } \\
\cline { 2 - 3 } & Water & Ethanol \\
\hline CsEMIM & Ionogel (soft) & Ionogel (CsE) \\
CsBMIM & dilution & Ionogel (CsB) \\
\hline
\end{tabular}

When the ionogels were rinsed with an ethanol-water mixture they became more translucent, reducing their yellow color (Figure 1). The continuous rinsing of the gels gradually eliminated the ionic liquid from inside the gels. The ethanol concentration that was used allowed for keeping the ionogels volume without causing drastic swelling or shrinkage. In the subsequent fluid phase replacement with acetone, the CsE ionogels decrease $25 \%$ their volume, and in the case of CsB, the reduction was $42 \%$. Apparently, the chitosan chains underwent rearrangement within the formed network to a more stable configuration as a result of the interaction with acetone [23].
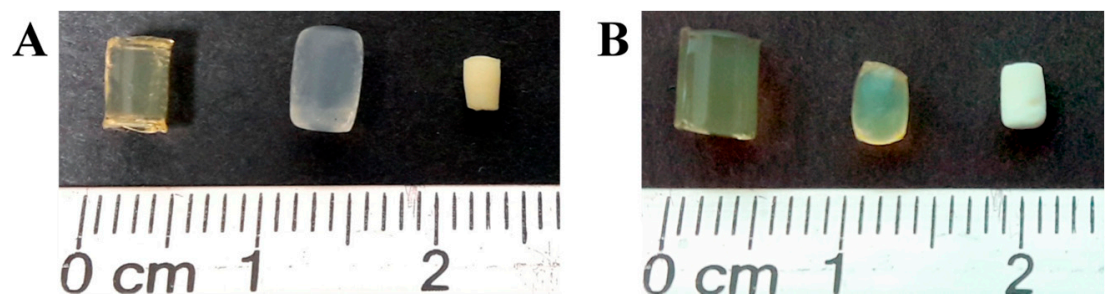

Figure 1. Materials formed from the Cs-IL solutions: (A) CsE and (B) CsB. From left to right: ionogel, acetogel and aerogel.

The obtained aerogels were rigid and brittle cylinders with opaque white color (Figure 1). The volume reduction when compared to the starting ionogels was of $73 \%$ for $\mathrm{CsE}$ and $82 \%$ for $\mathrm{CsB}$. This behavior is similar to that reported for aerogels obtained from chitosan and k-carrageenan [24]. Such volume reduction has been associated with the rearrangements of chitosan chains due to their lower affinity for acetone. This molecular movement does not cease until all of the acetone has been removed, even when the supercritical $\mathrm{CO}_{2}$ drying reduce the effects of surface tension in the material [25].

\subsection{Characterization of Chitosan Aerogels}

The infrared spectra $\left(2000-500 \mathrm{~cm}^{-1}\right)$ of chitosan and the obtained aerogels are shown in Figure 2. The main characteristic bands of chitosan are also observed in the aerogels spectra. At $1652 \mathrm{~cm}^{-1}$ is observed the stretching vibration band of the $\mathrm{C}=\mathrm{O}$ bond that is associated to the amide $\mathrm{I}$; the amide II $-\mathrm{NH}_{2}$ deformation is related to the band at $1580 \mathrm{~cm}^{-1}$; the band at $1424 \mathrm{~cm}^{-1}$ is associated to the $\mathrm{CH}_{2}$ bending; at $1380 \mathrm{~cm}^{-1}$, the symmetrical vibration deformation of the $\mathrm{CH}_{3}$ group is observed; the band at $1318 \mathrm{~cm}^{-1}$ is associated to the amide III; the antisymmetric tension mode of the COC bridge is observed at $1150 \mathrm{~cm}^{-1}$; finally, the fingerprint zone, between 1075 and $1026 \mathrm{~cm}^{-1}$, is characteristic of the polysaccharides [26,27]. There is no evident modification on the spectra that indicate chemical changes in the chitosan as result of the gel formation or the drying process. Furthermore, there are not absorbance bands that could be related to the presence of residual IL in the aerogels. 


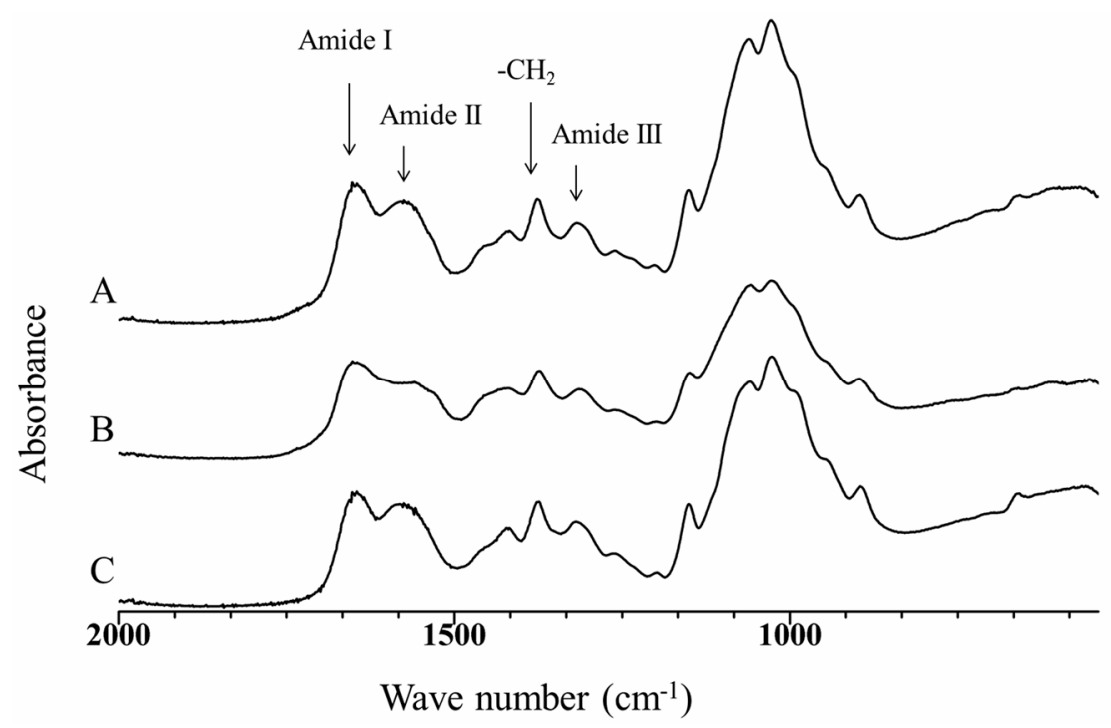

Figure 2. Fourier transform infrared spectrophotometer (FT-IR) spectra of (A) CsE aerogel; (B) CsB aerogel and $(\mathbf{C})$ chitosan.

The $\mathrm{N}_{2}$ adsorption and desorption isotherms of both types of aerogels are classified as type IV according to the IUPAC conventions (Figure 3). The observed hysteresis of $\mathrm{N}_{2}$ desorption at high relative pressures is indicative of a mesoporous dry material [28]. The specific surface area $\left(S_{\mathrm{BET}}\right)$ and pore volume that are calculated from the adsorption and desorption isotherms are included in Table 2. The specific surface areas of the aerogels are in the higher rank when compared with other pure polysaccharide aerogels $[24,29,30]$. The pore size obtained was within the range of mesopores, which is characteristic of aerogels.
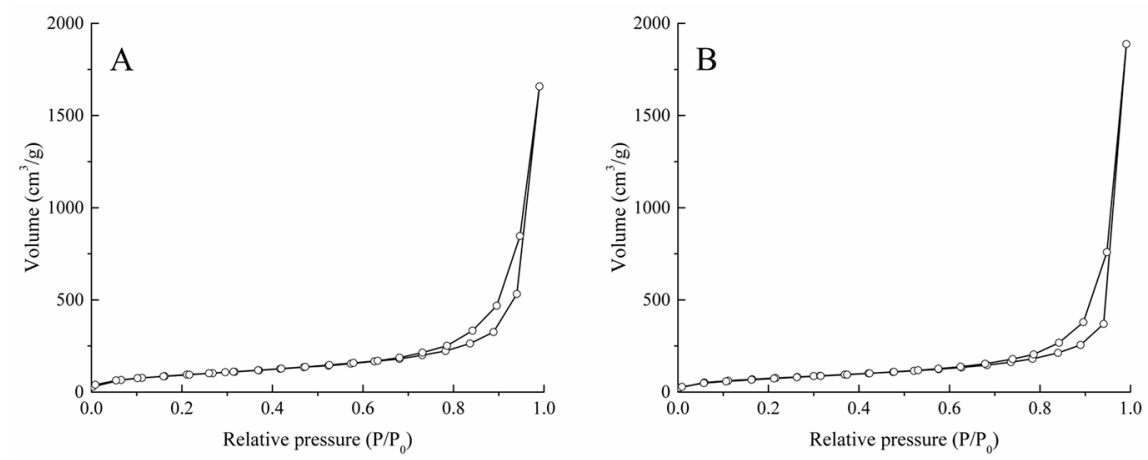

Figure 3. $\mathrm{N}_{2}$ adsorption and desorption BET isotherms of (A) CsE and (B) CsB aerogels.

Table 2. Specific surface area $\left(S_{\mathrm{BET}}\right)$, pore volume $\left(V_{\mathrm{p}}\right)$ and pore diameter $\left(D_{\mathrm{p}}\right)$ of the CsE and CsB aerogels.

\begin{tabular}{ccc}
\hline Parameters & CsE & CsB \\
\hline$S_{\mathrm{BET}}\left(\mathrm{m}^{2} / \mathrm{g}\right)$ & $358 \pm 79$ & $478 \pm 264$ \\
$V_{\mathrm{p}}\left(\mathrm{cm}^{3} / \mathrm{g}\right)$ & $0.0733 \pm 0.016$ & $0.236 \pm 0.083$ \\
$D_{\mathrm{p}}(\mathrm{nm})$ & $31.0 \pm 1.4$ & $46.0 \pm 3.6$ \\
\hline
\end{tabular}

The scanning electron microscopy (SEM) images of the aerogels are shown in Figures 4 and 5. Both of the aerogels appear as uniform materials with some imperfections, which could be caused by fracture events. At the highest magnification available $(\times 10,000)$, the internal structure of the aerogels looks as aggregated clumps forming a compact network with heterogeneous pores. The appearance of 
the pores is consistent with the mesoporous characteristics of the aerogels. The main difference between both types of aerogels is that the internal structure of CsB appears to be denser. The internal structure of the aerogels produced from ionogels is different to previously reported chitosan aerogels [24,29]. These differences could be related to the solvent-polymer interaction. Electrostatic repulsions dominate in the aqueous acid chitosan solutions. In such conditions, the polysaccharide molecules adopt an extended hydrodynamic volume conformation [17]. Conversely, these repulsive forces are absent in Cs-IL solutions; thus, the chitosan molecules have relatively smaller dimensions, generating more compact structures in their aerogels.

A

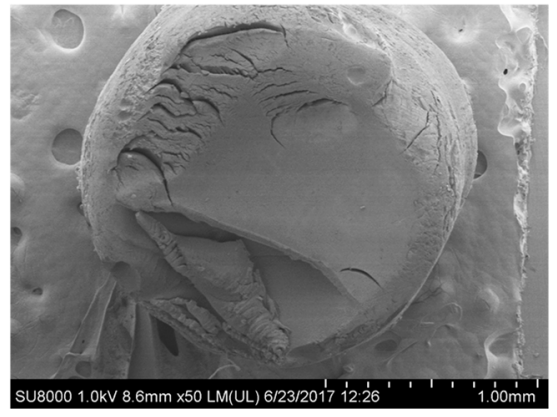

$\mathrm{C}$

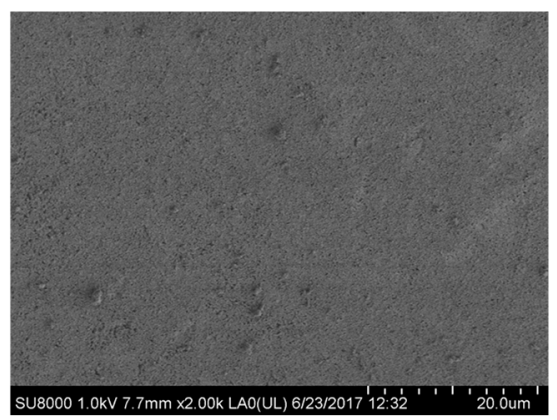

$\mathrm{B}$

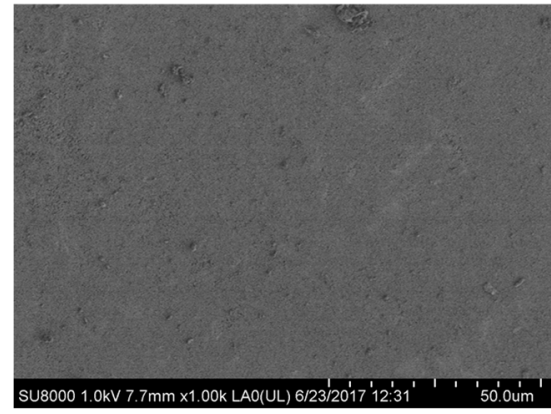

$\mathrm{D}$

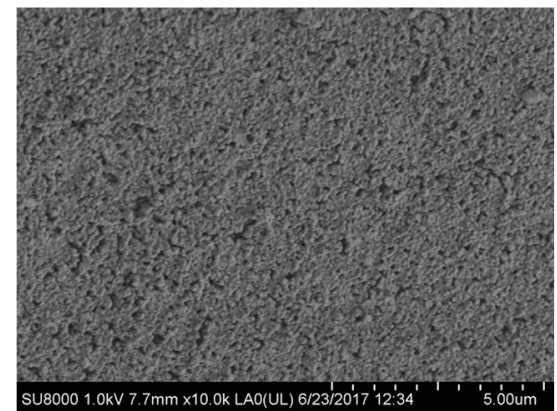

Figure 4. SEM images of the CsE aerogels (A) 50×; (B) 1000×; (C) 2000×; and, (D) 10,000× magnification.

A

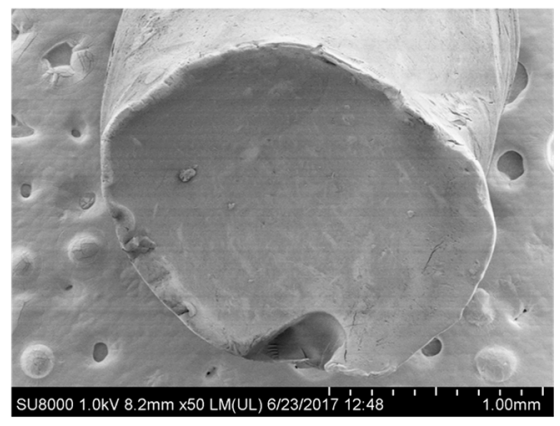

$\mathrm{C}$

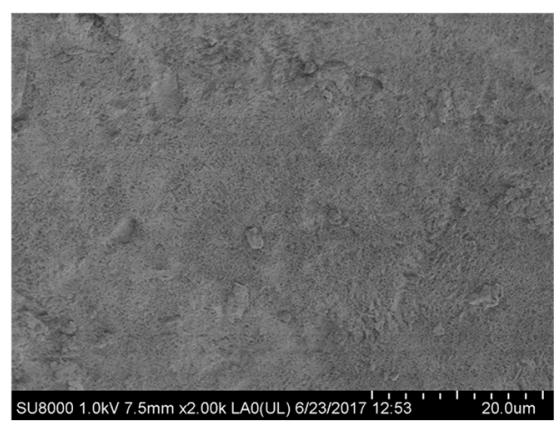

$\mathrm{B}$

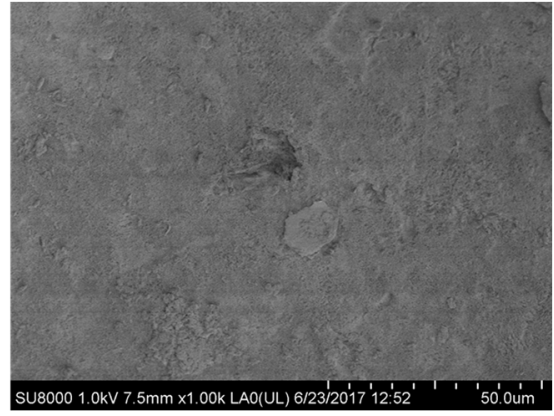

D

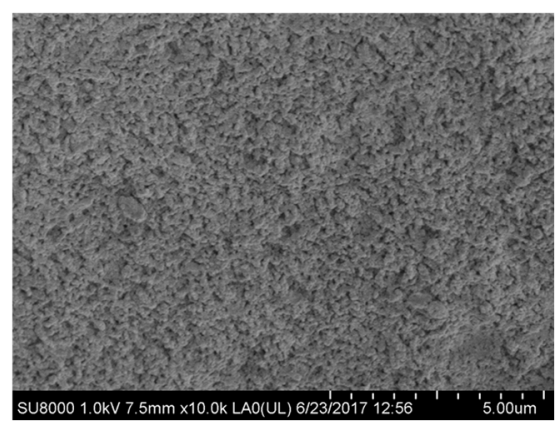

Figure 5. SEM images of the CsB aerogels. (A) 50×; (B) 1000×; (C) 2000×; and, (D) 10,000× magnification. 


\subsection{Diffusion Properties of Aerogels}

The physical characteristics of aerogels, such as their large surface area and mesoporosity, endow these materials with a large capacity to adsorb certain compounds. The diffusion properties are key to evaluating the performance of these materials for important applications in pharmacy and biotechnology, among others. For this purpose, dried aerogels that are obtained from chitosan solutions in EMIMAc and BMIMAc, as described previously, were swollen in water at different temperatures between 20 and $40{ }^{\circ} \mathrm{C}$ and their kinetics was followed. In Figures 6 and 7, the experimental data is presented.

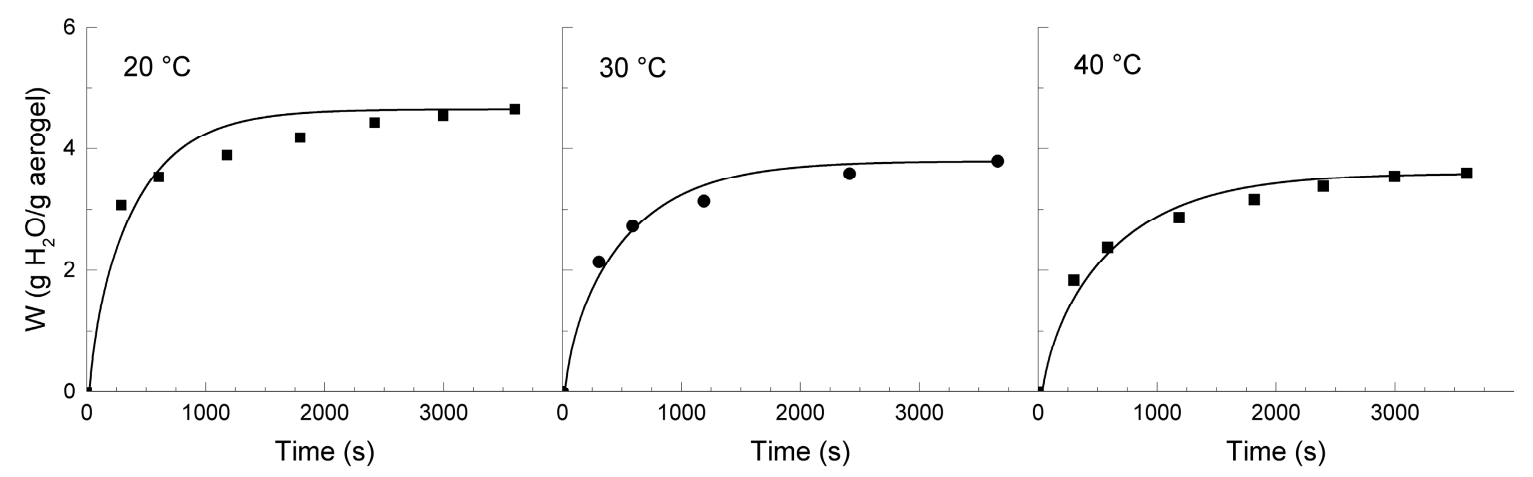

Figure 6. Swelling curves of the CsE aerogels in water at different temperatures $\left(20,30\right.$, and $\left.40{ }^{\circ} \mathrm{C}\right)$. Experimental data (points) and adjustment (lines) with Equation (2) are included.

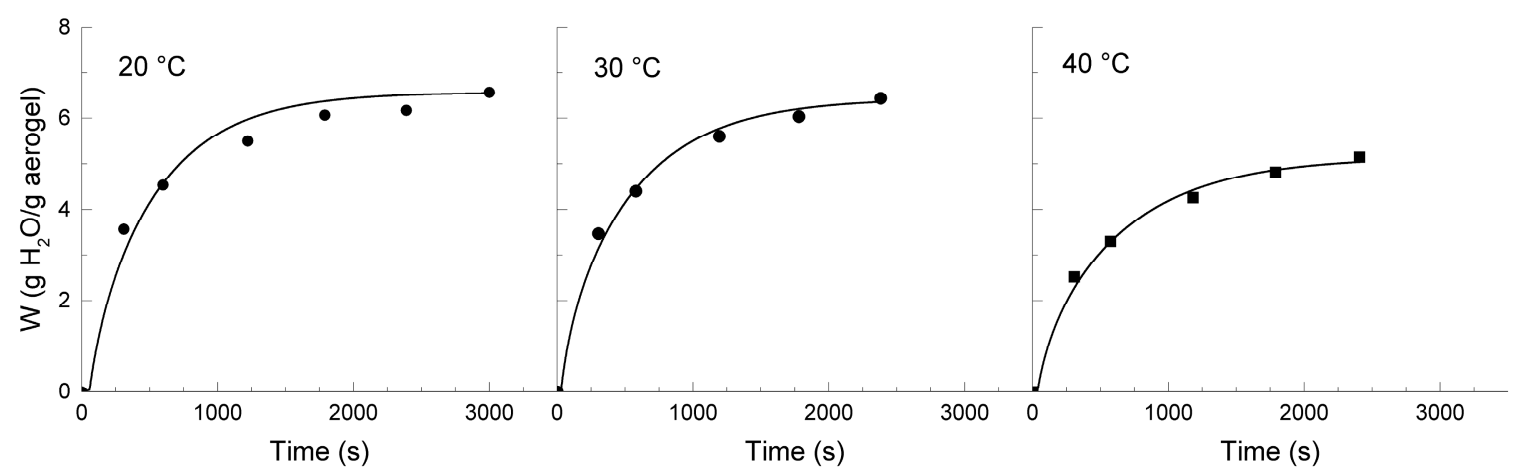

Figure 7. Swelling curves of the CsB aerogels in water at different temperatures $\left(20,30\right.$, and $\left.40{ }^{\circ} \mathrm{C}\right)$. Experimental data (points) and adjustment (lines) with Equation (2) are included.

The CsE aerogels absorbed between three and four times their weight and exhibited a decreasing swelling capacity with an increasing temperature. In contrast, the CsB aerogels showed a greater capacity of absorption (between five and six times their weight), and the effect of temperature on the swelling capacity seems to be less marked. When compared with the aerogels of chitosan that was obtained from aqueous acid solutions, the aerogels from chitosan in IL showed higher $W_{\infty}$. Previous studies with chitosan or chitosan-polyelectrolyte complex matrices that were prepared from aqueous media have shown a similar tendency to decrease the swelling capacity with temperature [20,31].

The Fick's law equation resolved for diffusion through a circular cylinder of radius $r$, keeping the diffusant concentration constant, becomes [32]:

$$
\frac{W}{W_{\infty}}=1-\sum_{n=1}^{\infty} \frac{4}{r^{2} \alpha_{n}^{2}} \exp \left(-D \alpha_{n}^{2} t\right)
$$

in which $W$ is the swelling degree at time $t$, and $W_{\infty}$ is the corresponding quantity at equilibrium, $\alpha_{n}^{2}$ are the $n$ first positive roots of the Bessel function of the first-kind, and $D$ is the diffusion coefficient. 
Equation (3) was solved for the first 15 terms of the summation and the diffusion coefficients that were adjusted through a non-linear least square fitting process. In all situations, satisfactory adjustments were obtained from Equation (2), as could be appreciated from the curves that are traced in Figures 6 and 7. The estimated values of $D$ are summarized in Table 3. The diffusion coefficients are similar for both types of aerogels and compare to those that are reported for swelling of other polymeric materials $[20,33]$. It should be remarked, however, the unusual trend that exhibits the diffusion coefficient, decreasing with the increase in temperature.

Table 3. Values of the degree of swelling, diffusion coefficient, second-order kinetic constant and the release exponent of CsE and CsB aerogels of swelled in water at different temperatures.

\begin{tabular}{|c|c|c|c|c|c|c|c|c|}
\hline \multirow[b]{2}{*}{$T\left({ }^{\circ} \mathrm{C}\right)$} & \multicolumn{4}{|c|}{ CsE } & \multicolumn{4}{|c|}{ CsB } \\
\hline & $\begin{array}{c}W_{\infty} \\
\text { (g } \mathrm{H}_{2} \mathrm{O} / \mathrm{g} \text { gel) }\end{array}$ & $\begin{array}{c}D \times 10^{10} \\
\left(\mathrm{~m}^{2} / \mathrm{s}\right)^{a}\end{array}$ & $\begin{array}{l}k \times 10^{4} \\
\left(s^{-1}\right)^{b}\end{array}$ & $n^{\mathrm{c}}$ & $\begin{array}{c}W_{\infty} \\
\text { (g } \mathrm{H}_{2} \mathrm{O} / \mathrm{g} \text { gel) }\end{array}$ & $\begin{array}{c}D \times 10^{10} \\
\left(\mathrm{~m}^{2} / \mathrm{s}\right)^{a}\end{array}$ & $\begin{array}{l}k \times 10^{4} \\
\left(s^{-1}\right)^{b}\end{array}$ & $n^{\mathrm{c}}$ \\
\hline 20 & 4.64 & 3.99 & 3.011 & 0.16 & 6.56 & 3.36 & 1.111 & 0.26 \\
\hline 25 & 3.96 & 3.55 & 3.426 & 0.20 & 5.22 & 3.32 & 1.367 & 0.31 \\
\hline 30 & 3.79 & 3.01 & 3.464 & 0.23 & 6.43 & 3.09 & 1.703 & 0.35 \\
\hline 40 & 3.59 & 2.54 & 4.481 & 0.29 & 5.15 & 2.68 & 2.738 & 0.37 \\
\hline
\end{tabular}

${ }^{a}$ Evaluated according to Equation (2), ${ }^{b}$ Evaluated according to Equations (8) and (10), ${ }^{c}$ Dimensionless release exponent from the power law relation.

It is well known that diffusion coefficients have a dependence on temperature that is similar to the Arrhenius equation:

$$
D=D_{0} \exp \left(-\frac{E_{\mathrm{D}}}{R T}\right)
$$

where $E_{\mathrm{D}}$ is the apparent activation energy for the diffusion process. For both aerogels, the expected linear dependence was obtained, but as a result of the inverse tendency that is shown by the diffusion coefficients with the temperature (Table 3), negative activation energy values were obtained. From a physical point of view, this fact indicates that there is another process competing with the diffusion, giving rise to negative values of this parameter. On the other hand, an analysis of the swelling values as a function of time, for each material at each temperature, according to the power law relation $W=k t^{n}$, allowed for finding the values of the release exponent, $n$, which are shown in the Table 3. Here, again, values are obtained that have no physical sense (for cylinders, $n$ should be between 0.46 and 1) [34]. As seen above, both of the aerogels have a porous structure, with pore sizes ranging between 15 and $23 \mathrm{~nm}$. According to these morphological characteristics, it should be expected that a Fickean-type diffusion kinetics would be fulfilled, since the relaxation of the polymer chains is not the limiting step for swelling. Consequently, the experimental data had to show a linear dependence between the swelling and $t^{1 / 2}$. However, this is not the case either.

All of this analysis shows that along with the diffusion, another process besides swelling is taking place. In this sense, the Eyring equation $[35,36]$ can provide information about the diffusion mechanism that takes place and sheds light on the causes of the observed negative values for the activation energies of the global diffusion process:

$$
k=\frac{k_{\mathrm{B}} T}{h} \exp \left(-\frac{\Delta G^{\ddagger}}{R T}\right)
$$

here $k_{\mathrm{B}}, h$, and $R$ are the Boltzmann, Planck, and the gas constants, respectively. The activation Gibbs free energy, $\Delta G^{\ddagger}$, is related to the activation enthalpy and entropy $\left(\Delta H^{\ddagger}, \Delta S^{\ddagger}\right)$, according to the following expression:

$$
\Delta G^{\ddagger}=\Delta H^{\ddagger}-T \Delta S^{\ddagger}
$$


Then, substituting (5) in (4):

$$
k=\frac{k_{\mathrm{B}} T}{h} \exp \left(\frac{\Delta S^{\ddagger}}{R}\right) \exp \left(-\frac{\Delta H^{\ddagger}}{R T}\right)
$$

which can be linearized as follows:

$$
\ln \frac{k}{T}=\ln \frac{k_{\mathrm{B}}}{h}+\frac{\Delta S^{\ddagger}}{R}-\frac{\Delta H^{\ddagger}}{R} \cdot \frac{1}{T}
$$

This kinetic treatment describes the dependence of the rate of a chemical reaction with temperature when the concepts of statistical mechanics are applied. Even though this analysis is based on the theory of absolute reaction rates developed to treat ordinary chemical reactions, it was demonstrated that this model could be successfully used to describe the kinetic treatment of viscosity and diffusion [37,38].

In order to elucidate the mechanism that takes place during the diffusion of water through these aerogels using the statistical approach of Eyring, it is necessary to estimate the rate constants of the swelling process. The experimental data showed an excellent fit to the following Equation proposed by Schott [39]:

$$
\frac{t}{W}=A+B t
$$

which describes a second-order kinetics with respect to the unrealized swelling:

$$
\frac{d W}{d t}=k\left(W_{\infty}-W\right)^{2}
$$

and the kinetic constant becomes equal to:

$$
k=\frac{1}{A W_{\infty}^{2}}
$$

The calculated values of the second-order rate constant, $k$, are included in Table 3, and were used to perform a thermo-kinetic analysis according to the Eyring Equation (7), as shown in Figure 8. The excellent adjustment that is obtained is evident. In Table 4 , the values of $\Delta H^{\ddagger}, \Delta S^{\ddagger}$, and $\Delta G^{\ddagger}$ are summarized.

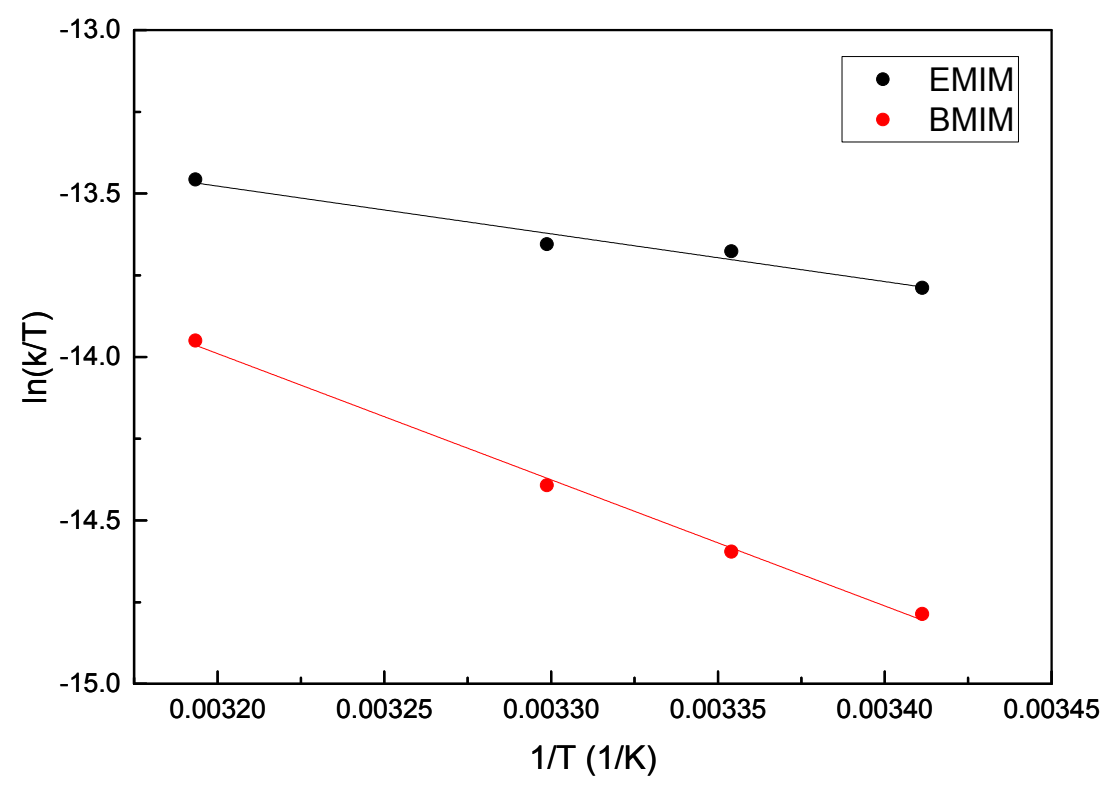

Figure 8. Plots of $\ln (k / T)$ versus $1 / T$ using the second-order rate constants obtained from Equation (10) for the two aerogels. 
Table 4. Activation energies calculated from $D$ by the Arrhenius Equation (3). Thermo-kinetic parameters: $\Delta H^{\ddagger}$ and $\Delta S^{\ddagger}$ estimated from the Eyring Equation (7) and $\Delta G^{\ddagger}$ calculated at $298.15 \mathrm{~K}$ using the Equation (5).

\begin{tabular}{|c|c|c|c|c|c|}
\hline Aerogel & Ea $(\mathrm{kJ} / \mathrm{mol})$ & $\Delta H^{\ddagger}(\mathrm{kJ} / \mathrm{mol})$ & $\Delta S^{\ddagger}(\mathrm{J} / \mathrm{mol} \cdot \mathrm{K})$ & $-T \Delta S^{\ddagger a}(\mathrm{~kJ} / \mathrm{mol})$ & $\Delta G^{\ddagger \mathrm{a}}(\mathrm{kJ} / \mathrm{mol})$ \\
\hline CsE & -17.5 & 12.1 & -271 & 80.7 & 92.9 \\
\hline CsB & -9.2 & 32.1 & -211 & 63.0 & 95.1 \\
\hline
\end{tabular}

When analyzing the thermo-kinetic parameters, it is observed that the activation enthalpies are positive due to the endothermic change from starting intermediates to the transition state during the overall diffusion process. According to these activation parameters, at $298.15 \mathrm{~K}$, the change of the activation Gibbs free energy is largely controlled by the activation entropy term (e.g., $-T \Delta S^{\ddagger}=81 \mathrm{~kJ} / \mathrm{mol}$ for the $\mathrm{CsE}$ aerogel), while the contribution of the activation enthalpy to the transition state is not so significant $\left(\Delta H^{\ddagger}=12 \mathrm{~kJ} / \mathrm{mol}\right)$. In this sense, it is important to note that these data indicate a significant increase in the order of the atoms that are involved during the pass to the transition state, with a small absorption of heat.

The values of these activation parameters are very similar to those that are observed during the formation of hydrogen bonds [40-42]. Their magnitudes coincide with the chemical nature of hydrogen bonding. Their formation involves relatively low energy and implicates the rearrangement of water molecules around the polymer, creating a hydration shell that is spatially oriented towards the hydroxyl and amino groups of chitosan.

At this point, it is important to remember that during the dissolution of chitosan in ionic liquids, the intra- and intermolecular hydrogen bonds of chitosan chains are broken. Although at the moment of forming the physical cross-linking the formation of some amount of hydrogen bonds is propitiated, these are relatively few in comparison with the large number of groups that are able to form hydrogen bonds in the polymer. In this way, during the swelling of the aerogels in water, along with the diffusion process, the generation of a huge amount of hydrogen bonds takes place with the consequent formation of a hydration shell. This analysis unambiguously supports the observed complex nature of the swelling processes of these aerogels, whose hydrated structure is apparently organized through hydrogen bonds. For this reason, a large contribution of $\Delta S^{\ddagger}$ to the transition state is appreciated, when compared to that of $\Delta H^{\ddagger}$.

\section{Conclusions}

Physical ionogels were obtained from chitosan solutions in EMIMAc and BMIMAc by non-solvent agent vapor diffusion (ethanol or water). The gels that were formed with ethanol treatment were rigid and brittle. Conversely, water vapor treatment only produce gels from the EMIMAc solution, these gels were too soft and brittle to be processed. Aerogels were produced by supercritical $\mathrm{CO}_{2}$ drying using ionogels that were produced by ethanol treatment. Such aerogels were low density mesoporous materials with surface area between 350 and $480 \mathrm{~m}^{2} / \mathrm{g}$. There is neither spectroscopic evidence of changes in the chemical identity of the chitosan after aerogels production nor evidence of residual IL in the obtained materials. The internal structures of both types of aerogels were similar, appearing as mesoporous materials that are formed by agglomerated clumps.

The swelling of these aerogels followed a second-order kinetics. The application of the Eyring equation to the dependence of the rate constants on temperature allowed for clarifying the characteristics of the diffusion of water. The change of the activation Gibbs energy is mainly controlled by the activation entropy, rather than by the activation enthalpy at the tested temperatures. The kinetics of swelling seems to be controlled by the formation of hydrogen bonding, instead of the diffusion of water itself. 
Aerogels with such characteristics have the potential for applications in the pharmaceutical industry as materials for the encapsulation, retention, and transport of model molecules that have affinities for chitosan. In the environmental field, these aerogels can be used as materials for the removal of pollutants from the effluents of industries in an extractive process by picking up minerals or compounds that are considered to be contaminants with affinity for chitosan. Another potential use is in the food industry or biotechnology, where components such as enzymes, proteins, or compounds that are part of a process could be immobilized inside the aerogels.

Acknowledgments: The authors want acknowledge the financial support of CONACYT through the Project CB-2011-01-169626 and the fellowship DC 2010-778 for GSL. Similarly, it is recognized the valuable input of the technical staff of the Biopolymer Research Group of CIAD.

Author Contributions: Jaime Lizardi-Mendoza and Waldo Argüelles-Monal conceived and designed the experiments; Gonzalo Santos-López performed the experiments; Gonzalo Santos-López, Waldo Argüelles-Monal and Jaime Lizardi-Mendoza analyzed the data; Maricarmen T. Recillas-Mota, Yolanda L. López-Franco and Elizabeth Carvajal-Millan contributed with reagents, materials and analytical advice; Gonzalo Santos-López, Waldo Argüelles-Monal and Jaime Lizardi-Mendoza wrote the paper and all the authors contributed to discuss and review the paper.

Conflicts of Interest: The authors declare no conflict of interest. The founding sponsors had no role in the design of the study; in the collection, analyses, or interpretation of data; in the writing of the manuscript, and in the decision to publish the results.

\section{References}

1. Valentin, R.; Bonelli, B.; Garrone, E.; Di Renzo, F.; Quignard, F. Accessibility of the Functional Groups of Chitosan Aerogel Probed by FT-IR-Monitored Deuteration. Biomacromolecules 2007, 8, 3646-3650. [CrossRef] [PubMed]

2. Pohako-Esko, K.; Bahlmann, M.; Schulz, P.S.; Wasserscheid, P. Chitosan Containing Supported Ionic Liquid Phase Materials for $\mathrm{CO}_{2}$ Absorption. Ind. Eng. Chem. Res. 2016, 55, 7052-7059. [CrossRef]

3. Guyomard-Lack, A.; Buchtová, N.; Humbert, B.; Bideau, J.L. Ion segregation in an ionic liquid confined within chitosan based chemical ionogels. Phys. Chem. Chem. Phys. 2015, 17, 23947-23951. [CrossRef] [PubMed]

4. Trivedi, T.J.; Rao, K.S.; Kumar, A. Facile preparation of agarose-chitosan hybrid materials and nanocomposite ionogels using an ionic liquid via dissolution, regeneration and sol-gel transition. Green Chem. 2014, 16, 320-330. [CrossRef]

5. Ma, B.; Li, X.; Qin, A.; He, C. A comparative study on the chitosan membranes prepared from glycine hydrochloride and acetic acid. Carbohydr. Polym. 2013, 91, 477-482. [CrossRef] [PubMed]

6. Kuzmina, O.; Heinze, T.; Wawro, D. Blending of Cellulose and Chitosan in Alkyl Imidazolium Ionic Liquids. ISRN Polym. Sci. 2012, 2012, 251950. [CrossRef]

7. Xiong, Y.; Wang, H.; Wu, C.; Wang, R. Preparation and characterization of conductive chitosan-ionic liquid composite membranes. Polym. Adv. Technol. 2012, 23, 1429-1434. [CrossRef]

8. Silva, S.S.; Santos, T.C.; Cerqueira, M.T.; Marques, A.P.; Reys, L.L.; Silva, T.H.; Caridade, S.G.; Mano, J.F.; Reis, R.L. The use of ionic liquids in the processing of chitosan/silk hydrogels for biomedical applications. Green Chem. 2012, 14, 1463-1470. [CrossRef]

9. Liu, Z.; Wang, H.; Liu, C.; Jiang, Y.; Yu, G.; Mu, X.; Wang, X. Magnetic cellulose-chitosan hydrogels prepared from ionic liquids as reusable adsorbent for removal of heavy metal ions. Chem. Commun. 2012, 48, 7350-7352. [CrossRef] [PubMed]

10. Naseeruteen, F.; Hamid, N.S.A.; Suah, F.B.M.; Ngah, W.S.W.; Mehamod, F.S. Adsorption of malachite green from aqueous solution by using novel chitosan ionic liquid beads. Int. J. Biol. Macromol. 2017. [CrossRef] [PubMed]

11. Muzzarelli, R.A.A. Biomedical Exploitation of Chitin and Chitosan via Mechano-Chemical Disassembly, Electrospinning, Dissolution in Imidazolium Ionic Liquids, and Supercritical Drying. Mar. Drugs 2011, 9, 1510-1533. [CrossRef] [PubMed]

12. Silva, S.S.; Mano, J.F.; Reis, R.L. Ionic liquids in the processing and chemical modification of chitin and chitosan for biomedical applications. Green Chem. 2017, 19, 1208-1220. [CrossRef] 
13. Silva, S.S.; Duarte, A.R.C.; Carvalho, A.P.; Mano, J.F.; Reis, R.L. Green processing of porous chitin structures for biomedical applications combining ionic liquids and supercritical fluid technology. Acta Biomater. 2011, 7 , 1166-1172. [CrossRef] [PubMed]

14. Kasaai, M.R. Various Methods for Determination of the Degree of N-Acetylation of Chitin and Chitosan: A Review. J. Agric. Food Chem. 2009, 57, 1667-1676. [CrossRef] [PubMed]

15. Ottøy, M.H.; Vårum, K.M.; Smidsrød, O. Compositional heterogeneity of heterogeneously deacetylated chitosans. Carbohydr. Polym. 1996, 29, 17-24. [CrossRef]

16. Scherließ, R.; Buske, S.; Young, K.; Weber, B.; Rades, T.; Hook, S. In vivo evaluation of chitosan as an adjuvant in subcutaneous vaccine formulations. Vaccine 2013, 31, 4812-4819. [CrossRef] [PubMed]

17. Rinaudo, M.; Pavlov, G.; Desbrières, J. Solubilization of Chitosan in Strong Acid Medium. Int. J. Polym. Anal. Charact. 1999, 5, 267-276. [CrossRef]

18. Montembault, A.; Viton, C.; Domard, A. Rheometric study of the gelation of chitosan in aqueous solution without cross-linking agent. Biomacromolecules 2005, 6, 653-662. [CrossRef] [PubMed]

19. Le Bideau, J.; Viau, L.; Vioux, A. Ionogels, ionic liquid based hybrid materials. Chem. Soc. Rev. 2011, 40, 907-925. [CrossRef] [PubMed]

20. Cárdenas, A.; Argüelles-Monal, W.; Goycoolea, F.M.; Higuera-Ciapara, I.; Peniche, C. Diffusion through Membranes of the Polyelectrolyte Complex of Chitosan and Alginate. Macromol. Biosci. 2003, 3, 535-539. [CrossRef]

21. Seddon, K.R. Ionic Liquids for Clean Technology. J. Chem. Technol. Biotechnol. 1997, 68, 351-356. [CrossRef]

22. Chen, Y.; Sun, X.; Yan, C.; Cao, Y.; Mu, T. The Dynamic Process of Atmospheric Water Sorption in [EMIM][Ac] and Mixtures of [EMIM][Ac] with Biopolymers and $\mathrm{CO}_{2}$ Capture in These Systems. J. Phys. Chem. B 2014, 118, 11523-11536. [CrossRef] [PubMed]

23. Vachoud, L.; Domard, A. Physicochemical properties of physical chitin hydrogels: Modeling and relation with the mechanical properties. Biomacromolecules 2001, 2, 1294-1300. [CrossRef] [PubMed]

24. Quignard, F.; Valentin, R.; Renzo, F.D. Aerogel materials from marine polysaccharides. New J. Chem. 2008, 32, 1300-1310. [CrossRef]

25. Subrahmanyam, R.; Gurikov, P.; Dieringer, P.; Sun, M.; Smirnova, I. On the Road to Biopolymer Aerogels-Dealing with the Solvent. Gels 2015, 1, 291-313. [CrossRef]

26. Brugnerotto, J.; Lizardi, J.; Goycoolea, F.M.; Argüelles-Monal, W.; Desbrieres, J.; Rinaudo, M. An infrared investigation in relation with chitin and chitosan characterization. Polymer 2001, 42, 3569-3580. [CrossRef]

27. Socrates, G. Infrared and Raman Characteristic Group Frequencies: Tables and Charts; John Wiley \& Sons: Chichester, NY, USA, 2004; ISBN 978-0-470-09307-8.

28. Sing, K.S.W.; Everett, D.H.; Haul, R.A.W.; Moscou, L.; Pierotti, R.A.; Rouquerol, J.; Siemieniewska, T. Reporting Physisorption Data for Gas/Solid Systems. In Handbook of Heterogeneous Catalysis; Wiley-VCH Verlag GmbH \& Co. KGaA: Weinheim, German, 2008; ISBN 978-3-527-61004-4.

29. Chang, X.; Chen, D.; Jiao, X. Chitosan-Based Aerogels with High Adsorption Performance. J. Phys. Chem. B 2008, 112, 7721-7725. [CrossRef] [PubMed]

30. Tsioptsias, C.; Stefopoulos, A.; Kokkinomalis, I.; Papadopoulou, L.; Panayiotou, C. Development of microand nano-porous composite materials by processing cellulose with ionic liquids and supercritical $\mathrm{CO}_{2}$. Green Chem. 2008, 10, 965-971. [CrossRef]

31. Goycoolea, F.M.; Fernández-Valle, M.E.; Aranaz, I.; Heras, Á. pH- and Temperature-Sensitive Chitosan Hydrogels: Swelling and MRI Studies. Macromol. Chem. Phys. 2011, 212, 887-895. [CrossRef]

32. Crank, J. The Mathematics of Diffusion, 2nd ed.; Clarendon Press: Oxford, UK, 1979; ISBN 0-19-853411-6.

33. Vázquez, B.; San Roman, J.; Peniche, C.; Cohen, M.E. Polymeric Hydrophilic Hydrogels with Flexible Hydrophobic Chains. Control of the Hydration and Interactions with Water Molecules. Macromolecules 1997, 30, 8440-8446. [CrossRef]

34. Ritger, P.L.; Peppas, N.A. A simple equation for description of solute release I. Fickian and non-fickian release from non-swellable devices in the form of slabs, spheres, cylinders or discs. J. Control. Release 1987, 5, 23-36. [CrossRef]

35. Eyring, H. The Activated Complex in Chemical Reactions. J. Chem. Phys. 1935, 3, 107-115. [CrossRef]

36. Wynne-Jones, W.F.K.; Eyring, H. The Absolute Rate of Reactions in Condensed Phases. J. Chem. Phys. 1935, 3, 492-502. [CrossRef] 
37. Eyring, H. Viscosity, Plasticity, and Diffusion as Examples of Absolute Reaction Rates. J. Chem. Phys. 1936, 4, 283-291. [CrossRef]

38. Kincaid, J.F.; Eyring, H.; Stearn, A.E. The Theory of Absolute Reaction Rates and its Application to Viscosity and Diffusion in the Liquid State. Chem. Rev. 1941, 28, 301-365. [CrossRef]

39. Schott, H. Swelling kinetics of polymers. J. Macromol. Sci. Part B 1992, 31, 1-9. [CrossRef]

40. Pauling, L. The Nature of the Chemical Bond and the Structure of Molecules and Crystals: An Introduction to Modern Structural Chemistry; Cornell University Press: Ithaca, NY, USA, 1960; ISBN 978-0-8014-0333-0.

41. Prabhumirashi, L.S.; Jose, C.I. Infra-red studies and thermodynamics of hydrogen bonding in ethylene glycol monoalkyl ethers. Evidence for a ten membered ring dimer. J. Chem. Soc. Faraday Trans. 2 Mol. Chem. Phys. 1975, 71, 1545-1554. [CrossRef]

42. Ishizuka, T.; Ohzu, S.; Kotani, H.; Shiota, Y.; Yoshizawa, K.; Kojima, T. Hydrogen atom abstraction reactions independent of $\mathrm{C}-\mathrm{H}$ bond dissociation energies of organic substrates in water: Significance of oxidant-substrate adduct formation. Chem. Sci. 2014, 5, 1429-1436. [CrossRef]

(C) 2017 by the authors. Licensee MDPI, Basel, Switzerland. This article is an open access article distributed under the terms and conditions of the Creative Commons Attribution (CC BY) license (http:/ / creativecommons.org/licenses/by/4.0/). 